\title{
Hacia un modelo de gestión de las organizaciones no lucrativas orientado a la excelencia
}

\section{Towards a model of management of non-profit organizations oriented to excellence}

Mora Ticó, Pere, ${ }^{1}$ Ph.D, García Oliva, Montserrat, ${ }^{2}$ Ph.D, Morquillas Gallego, Jordi, ${ }^{3}$ M.Sc.

Universidad Ramón Llull. España. 1Doctor en Geografía. Docente/investigador. pmora@peretarres.org. ${ }^{2}$ Doctora en Antropología social y Cultural. Docente/investigadora. mgarcia2@peretarres.org. ${ }^{3}$ Licenciado en Psicología. Doctorando en Ciencias de la Educación y del Deporte. jmorqui@peretarres.org

Recibido: 14-07-2016; Aceptado: 24-10-2016

\section{RESUMEN}

El objetivo de este artículo de reflexión es poner de manifiesto que las organizaciones no lucrativas deben desarrollar un modelo de gestión eficaz ante los retos a los que se enfrenta nuestra sociedad como el envejecimiento demográfico, el aumento de las desigualdades sociales y el incremento de la inmigración. El artículo analiza el impacto de la crisis económica y social en el sector no lucrativo e incluye: la necesidad de una estructura organizativa orientada a la eficiencia, la innovación y el liderazgo; la importancia de la interacción entre las organizaciones no lucrativas, empresas privadas y administraciones públicas (sociedad relacional); la incorporación del trabajo en red; la implementación de una política de transparencia y rendición de cuentas; la necesidad de asegurar la sostenibilidad económica y social mediante un modelo de gestión basado en la dirección estratégica, el marketing de servicios y una política de captación de fondos diversificada; y por último, se describen las competencias profesionales asociadas al liderazgo en organizaciones sociales.

Palabras clave: competencia profesional, emprendimiento social, impacto social, modelo de gestión, organizaciones no lucrativas, planificación estratégica. 
Mora et al. Hacia un modelo de gestión no lucrativas orientado a la excelencia

\begin{abstract}
The objective of this article of reflection is to show that non-profit organizations must develop an effective management model before the challenges that our society is facing, such as the aging of the population, the increase of social inequalities and the increase of immigration. The paper analyzes the impact of the economic and social crisis on the non-profit sector and includes: the need for an organizational structure oriented towards efficiency, innovation and leadership; the importance of interaction between non-profit organizations, private companies and public administrations (relational society); the incorporation of networking; the implementation of a policy of transparency and accountability; the need to ensure economic and social sustainability through a management model based on strategic management, service marketing and a diversified fundraising policy; and, finally, the professional competences associated with leadership in social organizations are described.
\end{abstract}

Key words: management model, nonprofit organizations, professional competence, social entrepreneurship, social impact, strategic planning.

\section{INTRODUCCIÓN}

\section{El impacto de la crisis económica y social en las Organizaciones No Lucrativas}

Las organizaciones no lucrativas se enfrentan al reto de dar respuesta a los problemas sociales que ha provocado la crisis económica y que ha afectado a una gran parte de la población. Se ha consolidado un modelo de sociedad caracterizada por el incremento de las desigualdades sociales, la precarización de las condiciones laborales, el aumento de la tasa de desempleo, ${ }^{1}$ la crisis estructural del estado de bienestar y una progresiva privatización de los servicios sociales. Tal y como recoge el séptimo informe FOESSA sobre exclusión y desarrollo social en España, ${ }^{2}$ se está produciendo un proceso de polarización y fractura social debido a la intensificación y cronificación de la pobreza, agravado por la apuesta por parte de los poderes políticos de un modelo que se basa en el crecimiento económico, el debilitamiento de los

1. Según la Encuesta de Población Activa (EPA), con datos de marzo de 2016, la tasa de paro en España se sitúan en un $21 \%$. Instituto Nacional de Estadística (INE):

http://www.ine.es/dyngs/INEbase/es/operacion.htm?c=Estadistica C\&c

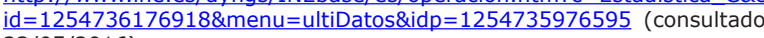
23/05/2016)

2. VII Informe FOESSA sobre exclusión y desarrollo social en España: http://www.foessa2014.es/informe/uploaded/descargas/VII INFORME. pdf (consultado 23/05/2016) servicios públicos y la implementación de políticas de austeridad que ahondan en una redistribución de la riqueza cada vez más injusta.

El factor demográfico, caracterizado por un progresivo envejecimiento de la población y por el aumento de los flujos migratorios provocados tanto por conflictos bélicos, como por motivos económicos o ambientales, tendrá una influencia clave en estos cambios de la estructura social en España. Según las previsiones de población a largo plazo del período 2012-2052, publicados por el Instituto Nacional de Estadística (INE), ${ }^{3}$ si se mantiene en un futuro la actual tendencia demográfica se producirá una pérdida progresiva de habitantes en las próximas décadas, previéndose para el año 2022 un 2,5\% menos de población que en 2012 y un $10 \%$ menos en 2052 . $\mathrm{Si}$ se mantienen los actuales ritmos de reducción de la mortalidad por edad, en 2051, la esperanza de vida al nacimiento se situaría en 86,9 años en los varones y de 90,7 en las mujeres. Estos datos nos sitúan en un escenario de futuro en el que se incrementa la tasa de dependencia, ${ }^{4}$ que alcanzaría un $99,54 \%$ en 2052, respecto a la

3. Parámetros de evolución demográfica INE 2012-2015:

http: //www.ine.es/jaxi/menu.do?type =pcaxis\&path $=/ \mathrm{t} 20$ p251/\&file=inebase (consultado 24/05/2016)

4. La tasa de dependencia incluye a los menores de 16 años y a los mayores de 64 años. 
del $50,39 \%$ de 2012 . Como se puede apreciar se trata de un contexto en que las variables económicas y sociales prevén una situación de progresivo empobrecimiento de una gran parte de la población junto a un aumento de la esperanza de vida combinado con una elevada tasa de dependencia.

Según el estudio La reacción del tercer sector social al entorno de la crisis, ${ }^{5}$ la crisis económica ha tenido una influencia negativa en el sector no lucrativo, principalmente a partir de 2010, en que los fondos públicos destinados a proyectos sociales se vieron reducidos en un $11 \%$ debido en gran medida a los recortes presupuestarios. Estos recortes en la financiación pública también han repercutido en el descenso de los recursos destinados por las empresas a políticas de responsabilidad social corporativa (RSC), al que habría que añadir la reestructuración del sector bancario y la conversión de las Cajas de Ahorro en Bancos. ${ }^{6}$ El impacto de la crisis ha producido cambios en la estructura de los recursos humanos de las organizaciones no lucrativas que vieron reducidas sus plantillas de forma significativa, pasando de un porcentaje del $41 \%$ de trabajadores profesionales en 2010 a un $29 \%$ en 2012. Este descenso se ha compensado con el incremento del voluntariado, que ha evolucionado desde un porcentaje del $59 \%$ en 2010 a un $71 \%$ en 2012 . La reestructuración del sector no lucrativo ha tenido un efecto negativo en la mayoría de entidades (el porcentaje de aquellas que se han extinguido se ha incrementado en un $16,6 \%$ entre 2008 y 2011) y en el aumento del índice de cese de actividad (incremento del $14,6 \%$ entre 2008 y 2011 ).

Este artículo recoge un análisis descriptivo de la situación actual de las organizaciones no lucrativas en función del contexto general existente, así como las perspectivas de futuro haciendo un

5. La reacción del Tercer Sector Social al entorno de la crisis: http://www.pwc.es/es/fundacion/assets/reaccion-3sector.pdf (consultado $24 / 05 / 2016$ )

6 . Este proceso de reestructuración del sector bancario ha significado un descenso del $24 \%$ en la financiación del Tercer Sector Social, teniendo en cuenta que en 2010 un 63,9\% de las Organizaciones No Lucrativas dependía de la financiación de las obras sociales y las cajas de ahorro. énfasis especial en el liderazgo de dichas organizaciones y en el desarrollo de las competencias de sus gestores.

\section{DISCUSIÓN}

\section{Hacia un modelo de gestión eficaz}

Ante un escenario de futuro, caracterizado por una situación socioeconómica desfavorable con unas perspectivas demográficas que tienden al envejecimiento progresivo de la población y al aumento de la tasa de dependencia, el sector no lucrativo deberá plantearse una reflexión interna que implicará una serie de acciones para dar respuesta a los nuevos retos a los que se enfrenta la sociedad, y cumplir, tal y como plantea Drucker (1996), el principal objetivo de las entidades sociales: provocar un cambio en las personas y en la sociedad.

Estas acciones incluyen: la redefinición de su misión y la transmisión de un mensaje claro sobre su papel ante los desafíos del entorno; generar un mayor impacto social a través de proyectos innovadores basados en la detección de las nuevas necesidades sociales emergentes; priorizar sus programas y proyectos, con el objetivo de optimizar sus recursos; conseguir la sostenibilidad financiera, superando el actual modelo de dependencia del sector público; potenciar el trabajo en red con otras entidades sociales y con los sectores privado y público; implicarse en la comunidad en diagnóstico, diseño y desarrollo de nuevos proyectos de intervención; implementar mecanismos que ayuden a medir el impacto social de sus actividades; mejorar los procesos de comunicación externa con el objetivo de concienciar y sensibilizar a la ciudadanía; y un replanteamiento de las competencias de sus profesionales, principalmente en el ámbito de la gestión, que configuren unas organizaciones flexibles y adaptadas a los continuos cambios del entorno. 
Mora et al. Hacia un modelo de gestión no lucrativas orientado a la excelencia

\subsection{Eficiencia, innovación $y$ liderazgo en las organizaciones no lucrativas}

Desde la perspectiva de la gestión, el sector no lucrativo deberá asumir el papel de agente innovador que, mediante el diseño e implementación de proyectos y servicios basados en la calidad y la eficiencia, pueda mejorar su impacto en la calidad de vida de las personas y colectivos en situación de exclusión social. Según Carreras, Iglesias y Sureda (2010), una organización no lucrativa es eficiente cuando consigue los objetivos propuestos mediante una adecuada optimización de los recursos invertidos (tanto humanos como económicos), así como de una apropiada administración y organización interna que les permite adaptarse a las nuevas realidades sociales y aprovechar las oportunidades que genera el entorno.

El objetivo principal de las organizaciones no lucrativas consiste en dar respuesta a necesidades sociales no atendidas, que ni el mercado ni el sector público han podido satisfacer, enfatizando su papel de agente innovador (Rodríguez Blanco, Carreras, Sureda, 2012). La innovación en las organizaciones no lucrativas implica un cambio en los procesos internos de gestión que favorece el trabajo en equipo, aumenta la capacidad de desarrollar propuestas y acciones por iniciativa propia de sus integrantes, genera un clima de confianza que favorece la capacidad de generar nuevas ideas, promueve el debate interno, asume riesgos que permiten la experimentación de nuevos servicios y proyectos y dispone del tiempo necesario para dedicarse a otras iniciativas más allá de la rutina. El objetivo final es promover el cambio y añadir valor y mejorar procesos, servicios o experiencias y que la organización se convierta en un lugar y propósito común de las ideas y de los equipos (Rodríguez Blanco, Carreras, Sureda, 2012).
Para que una organización no lucrativa alcance los objetivos de ser eficiente e innovadora necesita de un liderazgo transformador de la realidad, que luche contra las causas de las desigualdades a partir de una visión misional, de los valores de la propia organización y de la construcción de la legitimidad (Carreras, Leaverton y Sureda, 2009). Tal y como plantea Kotter (1996), el liderazgo es un proceso que tiene como objetivo dirigir y movilizar personas e ideas en una determinada dirección, por medios no coercitivos, e influir para que se produzcan los cambios adecuados que permitan crear el futuro. Las funciones del liderazgo deben establecer la directrices que ayuden a elaborar una visión de futuro compartida, así como las estrategias necesarias que permitan los cambios internos que faciliten su implementación, desarrollar instrumentos de comunicación interna que posibiliten la implicación de todas las personas que forman parte de la organización (profesionales y voluntarios), y transmitir la motivación y la inspiración necesaria para dotar de sentido el trabajo de los equipos y sus esfuerzos.

\subsection{Sociedad relacional $y$ responsabilidad social corporativa (RSC)}

La complejidad de la situación actual implica que la respuesta a los problemas sociales no puede plantearse únicamente desde el sector público, sino que debe implicar al resto de los agentes sociales: empresas, sector no lucrativo y universidades. Se trata de implementar un modelo de sociedad relacional en que todos los sectores aportan y construyen desde ámbitos compartidos e interrelacionados (Vidal, Torres, Guix y Peña, 2005). Según el anuario 2013 del Tercer Sector de Catalunya7 la interacción del sector no lucrativo con las administraciones públicas ha evolucionado y ha ido creciendo a medida que estas han desarrollado

7. Observatorio del Tercer Sector. Anuario del Tercer Sector Social de Catalunya: http://www.observatoritercersector.org/pdf/publicacions/OTS Anuario2013 doc sintesis cast.pdf (consultado 25/05/2016) 
una política de servicios sociales más amplia y compleja, caracterizada por una relación de proveedor cliente y una excesiva dependencia a nivel de financiación por parte de las entidades sociales. El modelo de colaboración deberá evolucionar hacia un sistema de corresponsabilidad en que la ciudadanía y las administraciones públicas participen conjuntamente en los procesos de detección de las necesidades sociales y que, sus destinatarios formen parte activa en su solución y en el diseño e implementación de los servicios sociales. Se trata de aprovechar la estructura y organización del sector público con las ventajas del sector no lucrativo en la prestación de los servicios sociales: proximidad al usuario, capacidad de iniciativa y de innovación y flexibilidad.

Los departamentos de investigación de las universidades pueden aprovechar la experiencia del sector no lucrativo para sistematizar un cuerpo teórico que permita incrementar sus potencialidades como agente clave en la mejora de la sociedad y trabajar en colaboración con otras organizaciones sociales, gobiernos y empresas (Goodman y Murillo, 2011). Las universidades deben fomentar titulaciones que puedan servir de contexto para la formación de los futuros directivos del sector sin ánimo de lucro y generar espacios de debate y reflexión en los que deben participar todos los agentes sociales, tanto públicos como privados.

El ámbito empresarial ha sido tradicionalmente un factor clave en la política de captación de fondos de las organizaciones no lucrativas. Los proyectos de responsabilidad social corporativa (RSC) de las empresas han significado un avance en la relación entre el sector privado y el no lucrativo y ha favorecido su implicación en la respuesta a los nuevos retos de una sociedad globalizada (Vernis, Iglesias, Sanz y Saz, 2004). La RSC es la responsabilidad que asume una empresa u organización ante la sociedad por los impactos de su actividad y por la corresponsabilidad en los asuntos comunes que afectan a la sostenibilidad del mercado, de la sociedad y del medio ambiente. ${ }^{8}$ Desde la perspectiva de la empresa la RSC implica un compromiso que incluye cinco grandes ámbitos de impacto empresarial que forman parte de su modelo de empresa y afecta a todas las áreas: económico, laboral, ambiental, social e integral. Según Vernis, Iglesias, Sanz y Saz (2004), los niveles de colaboración entre las empresas y las causas sociales pueden tener diversos grados de implicación. Por un lado, una empresa puede plantearse una colaboración puntual con una organización no lucrativa que implique donaciones a corto y medio plazo para proyectos sociales (modelo filantrópico); por otra parte, puede desarrollar un modelo de colaboración estratégico en el que, tanto la empresa como la organización no lucrativa suman esfuerzos en aquellos ámbitos en los que tienen intereses comunes y que puede concretarse en un proyecto con impacto social; finalmente, las empresas pueden plantearse integrar la RSC en toda su estrategia empresarial (trabajadores, clientes, proveedores y el resto de stakeholders).

En esta tercera fase la empresa y la organización no lucrativa comparten algunos valores y la visión de futuro sobre la organización y sobre el entorno y establecen relaciones continuadas a lo largo del tiempo dentro de su marco relacional (Lozano, 2005). La colaboración de las organizaciones no lucrativas con las empresas ha evolucionado, desde una relación basada en la financiación de sus proyectos, a un modelo colaborativo en que ambas entidades trabajan por un objetivo común (Abenoza, Carreras y Surera, 2015). Desde la perspectiva de la financiación, la RSC les permite a las organizaciones no lucrativas una mayor flexibilidad y diversificación en la captación de fondos: campañas de sensibilización con los empleados, aportación de bienes y servicios,

8. Responsabilidad Global: http://rsedefinicions.blogspot.com/2014/04/ responsabilitat-social-segons-josep.html 
Mora et al. Hacia un modelo de gestión no lucrativas orientado a la excelencia

promoción del voluntariado corporativo, compra de productos y servicios, cesión de espacios de comunicación, utilización de las redes de distribución de las empresas...

\subsection{Trabajo en red de las Organizaciones No Lucrativas}

Para poder hacer frente a los complejos retos de futuro a los que se enfrenta el sector no lucrativo éste debe estar cohesionado y coordinado. Tal y como apuntan Vernis, Iglesias, Sanz y Saz (2004), la aparición de problemáticas complejas, la creciente demanda de transparencia, la optimización de los recursos de que disponen, el reconocimiento del tercer sector como actor político y el desarrollo de las nuevas tecnologías como elemento clave de información y comunicación, hacen necesario el fomento de la cultura de la participación.

Las diferentes redes 0 estructuras de segundo nivel, en función de los objetivos de las entidades sociales, sirven para interlocutar con el sector público y obtener viabilidad en sus proyectos e influencia política. Son propias de las estructuras de segundo y tercer nivel y son especialmente importantes para ejercer de lobby de presión ante situaciones que se perciben como injustas. El trabajo en red permite desarrollar proyectos y programas de actuación que permiten dotarles de un sentido transversal e integrador, así como tener un efecto multiplicador de su eficacia e impacto social. También permite desarrollar espacios de encuentro en los que se pueden compartir proyectos, actividades y campañas de comunicación y sensibilización que potencian su visualización ante la sociedad; contribuir a la eficiencia y a la optimización de sus recursos; reforzar la sensación de pertenencia a la entidad y su papel institucional; y generar conocimiento, a través del intercambio de experiencias y de modelos de gestión innovadores.
El futuro del trabajo colaborativo entre organizaciones no lucrativas deberá plantearse desde la perspectiva estratégica mediante redes que apliquen las nuevas tecnologías que favorezcan su nivel de flexibilidad y de unión temporal, basándose en el principio de interactividad que facilita el intercambio de información y la compartición de proyectos.

\subsection{Transparencia y rendición de cuentas}

Tal y como plantean Vernis, Iglesias, Sanz y Saz (2004), las organizaciones no lucrativas tradicionalmente han rendido cuentas en el ámbito financiero ya que se trata de un requisito básico para el adecuado funcionamiento de la entidad y permite trabajar en la mejora de la eficacia y la optimización de los recursos de que disponen. Los principales mecanismos de transparencia económica que deben establecer las organizaciones no lucrativas pueden ser: poner a disposición de todos los integrantes de ellas la entidad el estado de cuentas y el balance, procurar que las actividades económicas no sean contrarias a su misión o finalidades sociales, establecer un código ético que regule las actividades económicas, y establecer un principio de sostenibilidad que implique la captación de recursos propios que permitan conseguir sus objetivos.

Para desarrollar una política de transparencia eficaz es necesario comunicar otros aspectos más allá de los económicos como: el nivel departicipación interna de profesionales y usuarios, la participación del voluntariado en la toma de decisiones, la tipología e impacto social de los proyectos y servicios, la adecuada detección de necesidades ante las situaciones cambiantes del entorno, la coherencia con la misión y los valores de la entidad, el diseño de los sistemas de información y control (incluidas las auditorías externas), y el nivel de comunicación de las relaciones y redes que se han establecido... 
Estas organizaciones deben asumir su responsabilidad estratégica en la política de transparencia y rendición de cuentas. Esta responsabilidad estratégica implica que el modelo de gestión debe definir claramente la visión de futuro de la entidad (con la finalidad de visualizar hacia donde se dirige y crear un proyecto compartido por todos sus miembros), la fijación de metas y objetivos claramente definidos, la existencia de un marco adecuado definido a nivel estratégico y operativo que facilite la toma de decisiones, y la evaluación de las estrategias y objetivos que permite a los equipos ser más efectivos y eficientes (Iglesias, Carreras y Sureda, 2010).

\subsection{La dirección estratégica de las organizaciones no lucrativas}

Para conseguir el objetivo de la excelencia en la prestación de servicios, las organizaciones no lucrativas debe desarrollar un sistema de gestión que tenga en cuenta los condicionantes del entorno (económicas, sociales, demográficas, políticas, legales, ambientales...), sus propias capacidades (puntos fuertes y aspectos a mejorar), la proximidad al usuario, una misión definida y consensuada, una visión clara de liderazgo, unos valores compartidos por todos los integrantes de la organización, una estructura organizativa descentralizada y participativa, y una cultura organizativa que genere confianza y fomente la implicación de los trabajadores y voluntarios. La responsabilidad de los directivos es tomar decisiones relativas a la estructura organizativa de la entidad, los sistemas de gestión de la calidad, la adecuada utilización de las tecnologías de la información, las políticas de recursos humanos, y los vínculos con otras organizaciones Daft (1998).

La continuidad y sostenibilidad de las entidades sociales necesita que estas sean capaces de desarrollar un sistema de gestión que se adapte a la compleja situación del entorno actual y que incorpore una actitud estratégica que impregne de forma transversal a todas las áreas de la entidad. Los principales instrumentos de gestión estratégica de que disponen las organizaciones sociales son la planificación estratégica, el marketing de servicios y las estrategias de captación de fondos.

\section{La planificación estratégica es un} proceso de diagnóstico, análisis, reflexión y toma de decisiones colectivas mediante el cual una organización define cuáles son sus principales retos en un determinado período de tiempo y fija sus estrategias y objetivos para conseguirlos (Burgwal y Cuellar, 1999). Un elemento fundamental en el proceso de planificación estratégica es la participación de los integrantes de la organización en la definición de la fase filosófica (misión, valores y visión de futuro) y en la toma de decisiones relacionadas con el diseño y la implementación de las estrategias y objetivos (Navajo, 2009). La evaluación y control del plan estratégico permitirá medir y valorar los resultados de las estrategias y objetivos planteados $y$, al mismo tiempo, establecer medidas correctoras en los indicadores de seguimiento que se han establecido previamente.

Según Kotler (1996), el Marketing es un mecanismo de gestión que tiene como objetivo identificar, anticipar y satisfacer las necesidades y motivaciones de los usuarios de una organización de una forma rentable y satisfactoria. En el caso de las organizaciones no lucrativas la rentabilidad va más allá de la económica, es social y comunitaria. Se trata de una metodología de trabajo que consiste en recoger, valorar y analizar la información necesaria sobre las necesidades de los usuarios y definir una cartera de servicios que se adapten a estas necesidades. Para evitar la homogeneización de los servicios, las entidades sociales tienen que definir qué ventaja competitiva desean implementar mediante la concreción de qué servicios y estrategias de atención a los usuarios 
Mora et al. Hacia un modelo de gestión no lucrativas orientado a la excelencia

dispone la entidad para diferenciarse de la competencia (posicionamiento) y la identificación de uno o diversos grupos de usuarios con necesidades homogéneas que justifiquen dirigirles una oferta de servicios específica y diferenciada (segmentación). A través del Plan de Marketing se establecen las políticas de comunicación (interna y externa), se fija la política de precios y los mecanismos de captación de fondos que permita asumir los costes de producción de estos servicios. La política de comunicación debe incrementar el nivel de sensibilización de la ciudadanía sobre el proyecto social de la organización no lucrativa mediante la máxima presencia en los medios de comunicación con el objetivo de difundir y visualizar su contribución a la sociedad.

La política de captación de fondos es un elemento fundamental para el desarrollo de los proyectos y servicios que prestan las entidades sociales y debe guiarse por unos principios éticos que regulen las campañas de comunicación, marketing y relaciones públicas. El principal objetivo es asegurar la sostenibilidad económica de la organización mediante una estrategia de diversificación que incluye la captación de fondos públicos y privados, así como aumentar la base social mediante la incorporación de socios y voluntarios. Diversificar significa minimizar el riesgo que supone la dependencia excesiva de una única fuente de financiación (tradicionalmente pública) en un contexto como el actual, en que las posibilidades de captar fondos son amplias debido a la incorporación de las empresas como un agente clave. Según Muñoz (2007), los principales recursos que tiene a su disposición el sector social son: públicos (subvenciones, convenios de colaboración, conciertos, contratación de servicios, cesión de usos de equipamientos públicos...), propios (cuotas de socios, grandes donantes, herencias y legados, campañas económicas puntuales, venta de servicios y productos), empresas (esponsorización, mecenazgo, marketing con causa, financiación de proyectos compartidos...), y otras entidades sociales (fundaciones privadas y de empresas, obras sociales de entidades financieras).

\section{Las competencias profesionales asociadas al liderazgo en organizaciones sociales}

Si las entidades sociales están inmersas en procesos de cambio de gestión, también los líderes que gestionan dichas entidades empiezan a desarrollar nuevas estrategias de dirección. Los líderes de este tipo de entidades han visto cómo su formación en un ámbito marcadamente técnico no les permite afrontar, de forma absoluta, la dirección en el marco de los nuevos modelos de gestión. Las competencias técnicas no son suficientes si se quiere liderar de manera efectiva un proyecto. El liderazgo de estas entidades debe desarrollar nuevas competencias profesionales que, hasta el momento, no estaban contempladas en un ámbito demasiado cercano al planteamiento cooperativo y asistencial.

Pero para presentar estos cambios en el campo de las competencias profesionales de liderazgo, en primer lugar se debe partir del concepto de competencia. El análisis de las competencias de liderazgo se ha ido desarrollando en los últimos años y existen, incluso, programas específicos de coaching (Cardona, 1999) y de diagnóstico y de desarrollo de estas (Cardona y García Lombardía, 2011; Cardona y García Lombardía, 2002). En un marco general, las competencias se asocian de manera causal al éxito en un determinado lugar de trabajo y se crean diccionarios que las clasifican y las desglosan en distintos niveles (Ayuntamiento de Barcelona, 2011; Alles, 2002). No es fácil definir competencia y es preciso remontarse a 1973 cuando McClelland empieza a utilizar dicho término para la asunción del liderazgo diferenciar los directivos sobresalientes de aquellos que, simplemente, hacen bien las 
cosas. Desde entonces se han originado distintas definiciones de competencia y son muchos los autores que han disertado sobre ellas (Boyatzis, 1982; Spencer y Spencer, 1993; Goleman, 2005 en Martínez-González, 2011). En este trabajo se adoptará la definición y el modelo de Cardona (1999) por ser uno de los más divulgados. Según el autor las competencias son: "comportamientos observables y habituales que posibilitan el éxito de una persona en su actividad o función" (p.20).

En el ámbito social ha existido una tendencia de huir de la gestión considerándola opuesta a la intervención. De alguna manera se asumía que si se era un buen técnico también se podía ser un buen directivo. Las distintas formaciones académicas con visión profesionalizadora preparan a los futuros profesionales de cada una de las disciplinas para ejercer de la mejor manera como buenos técnicos (buenos educadores, buenos trabajadores sociales, buenos pedagogos...). Durante la formación se trabajan las competencias necesarias que les llevarán a implementar, de manera óptima, el desarrollo de sus funciones cuando las apliquen en su trabajo cotidiano.

Ahora bien, cuando el profesional en el desempeño de sus tareas debe acceder a cargos directivos, de gestión o de coordinación se da cuenta que esas competencias técnicas que tanto le han servido para la atención directa a los beneficiarios, no son suficientes para dirigir o gestionar un equipo. ¿Qué espera de un directivo su equipo de trabajo? Desea que conozca claramente la misión de la entidad, que sepa construir una visión y que sea capaz de desarrollar estrategias para obtener el compromiso identitario. En definitiva, el equipo aspira que exista una visión de conjunto y una perspectiva clara y definida de futuro. Pero además, el equipo anhela, que el directivo sea capaz de conducirlo a través de alguno de los distintos estilos de liderazgo. Cuando se asume la responsabilidad de un equipo, debe realizarse seguimiento de este, analizar las distintas situaciones, gestionar conflictos, resolver problemas, tomar decisiones, delegar, motivar, liderar, etc. Así, la comunicación, el trabajo en equipo, la solución de problemas, el liderazgo del equipo y la conducción de reuniones se convertirán en herramientas necesarias para los directivos actuales.

Teniendo en cuenta que los conocimientos se quedan desfasados con rapidez, se valora más, en el desempeño profesional de las personas, su capacidad de aprendizaje y la adaptación a nuevas situaciones. En este marco es válido preguntarse ¿qué competencias debe tener un líder en general? y, más concretamente, ¿qué competencias debe tener un líder en las entidades sociales? Con base en el modelo de Cardona, (1999) sobre dimensiones o talentos del directivo (talento estratégico: capacidad de desarrollar e implementar estrategias que conduzcan a buenos resultados económicos; talento ejecutivo: capacidad para desarrollar a los colaboradores, adaptando las tareas a las aptitudes de cada uno y talento de liderazgo personal: capacidad de crear confianza e identificación de los colaboradores con la misión de la organización) es factible establecer las siguientes competencias directivas:

Unida al talento estratégico, la competencia de negocio (visión de negocio, visión de la organización, gestión de recursos, orientación al cliente, red de relaciones efectivas, negociación).

Unida al talento ejecutivo, la competencia interpersonal (resolución de conflictos, delegación, coaching, trabajo en equipo, comunicación)

Unida al talento de liderazgo personal la competencia personal (proactividad, autogobierno, gestión de personal, mejora personal).

Los modelos utilizados hasta el momento, sobre liderazgo y competencias se centraban en organizaciones con ánimo de lucro y en comparaciones entre este modelo de gestión y la gestión en el ámbito social. El estudio preliminar realizado por Carreras, Leaverton, y 
Mora et al. Hacia un modelo de gestión no lucrativas orientado a la excelencia

Sureda (2009), va un paso más allá y concreta una investigación centrada en el liderazgo en el ámbito social. Los autores pretenden analizar, por el momento, qué es el liderazgo de las organizaciones no lucrativas y no qué no es. En la revisión realizada sobre la literatura existente, los autores destacan como una de las competencias más valoradas la de la inteligencia emocional y cómo esta parece encontrarse más presente entre los líderes de las organizaciones sociales. Como conclusiones destacan también la importancia de la experiencia y la reflexión para reconocerse como un buen líder. Las competencias encontradas se han agrupado en las siguientes categorías ${ }^{9}$ :

- Liderazgo inspiracional: comunicación clara, confianza en sí mismo y coherencia.

- Inteligencia emocional: conocimiento de sí mismo y empatía.

- Visión para los negocios: visión para los negocios, pensamiento sistémico y reconocimiento de patrones.

- Liderazgo compartido y construcción de equipos motivados y autónomos: desarrollo de otros y trabajo en equipo y colaboración.

Con este estudio se completa una carencia. Qué es aquello que tienen las organizaciones no lucrativas a nivel competencial que las hace distintivas. Sin embargo, debe seguir indagándose y ampliando este tipo de estudios puesto que son todavía incipientes. También se debe seguir con las investigaciones comparativas para que el enriquecimiento resulte bilateral.

\section{CONCLUSIONES}

Para dar respuesta a las necesidades emergentes de los colectivos de personas en situación de riesgo de exclusión en un contexto social y económico complejo, las organizaciones sin ánimo de lucro deberán

9. Cabe destacar que la clasificación surge del análisis de las entrevistas realizadas a una pequeña muestra de directivos de relevantes entidades sociales como primera detección de competencias propias o más valoradas y que, dicho estudio, pretende ser ampliado con una muestra más amplia. incorporar instrumentos de gestión basados en la eficiencia, la innovación y en un liderazgo que facilite la motivación y la implicación de las personas en la misión y objetivos de la organización.

Un modelo de gestión eficaz implica: analizar las variables del entorno (sociales, económicas, demográficas, políticas, ambientales y tecnológicas); implementar mecanismos de recogida de información que potencien la colaboración intersectorial y el trabajo en red que impliquen a las empresas (mediante la responsabilidad social corporativa), las administraciones públicas, otras organizaciones nolucrativas, y a los propios usuarios (mediante su empoderamiento) en la detección de nuevas necesidades sociales, así como en el proceso de diseño e implementación de los servicios y proyectos que darán respuesta a estas necesidades (marketing de servicios); desarrollar un sistema de captación de fondos diversificado que asegure la sostenibilidad de las entidades sociales y la estabilidad de sus proyectos y desarrollar una política de transparencia y rendición de cuentas que incorpore la medición del impacto social de sus actividades.

Para conseguir estos objetivos el liderazgo que deben ejercer los directivos de las organizaciones no lucrativas deben incorporar nuevas competencias que unan al talento estratégico la competencia de negocio (basada en una visión integral de la gestión organizacional) y al talento de liderazgo personal la competencia personal (involucrar a las personas que forman parte de la entidad en la misión y la visión de futuro). Este modelo de liderazgo debe basarse en la implementación de un sistema que favorezca la participación interna de profesionales y voluntarios en la toma de decisiones, que facilite la construcción de equipos motivados e implicados en el proyecto común, así como la creación de espacios destinados a potenciar el emprendimiento interno y la innovación. 


\section{REFERENCIAS}

Abenoza, S., Carreras, I. y Surera, M. (2015). Colaboraciones ONG y empresas que transforman la sociedad. Barcelona: Instituto de Innovación Social de ESADE.

Alles, M. (2002) Gestión por competencias: el diccionario. Buenos Aires: Ediciones Granica.

Ayuntamiento de Barcelona (2011). Diccionario de competencias. Recuperable en: http://w27.bcn.cat/porta22/es/altres/ diccionari.jsp

Burgwal, G.; Cuellar, J.C. (1999). Planificación estratégica y operativa aplicada a gobiernos locales. Quito: Abya Ayala.

Cardona, P. (1999) En busca de las competencias directivas. En Revista de antiguos alumnos. (23):19-26. Recuperable en http://www.ee.iese.com/76/76pdf/ afondo2.pdf

Cardona, P. y García Lombardía, P. (2002) Cómo diagnosticar y desarrollar competencias. En Revista de antiguos alumnos: 14-18. Recuperable en: http:// www.sld.cu/galerias/pdf/sitios/infodir/ como diagnosticar competencias.pdf

Cardona, P. y García Lombardía, P. (2011). Cómo desarrollar las competencias de liderazgo. Pamplona: EUNSA

Carreras, I., Leaverton, A. y Sureda, M. (2009). Líderes para el cambio social. Características y competencias para el liderazgo en las ONG. Barcelona: Instituto de Innovación Social de ESADE.

Daft, R.L. (1998). Teoría y diseño organizacional. México: Thompson Editores.

Drucker, P. (1996). Dirección de instituciones sin fines de lucro. Barcelona: El Ateneo.

Goodman, J. y Murillo, D. (2011). Las diez innovaciones sociales más destacadas $e$ inspiradoras. Barcelona: Instituto de Innovación Social de ESADE.
Iglesias, M., Carreras, I. y Sureda, M. (2010). Eficiencia para el cambio social. ONG que mejoran su rendimiento. Barcelona: Instituto de Innovación Social de ESADE.

Kotler, P. (1996). Dirección de marketing. Barcelona: Prentice Hall.

Kotter, J.P. (1996). Leading change. Boston: Harvard Business School Press.

Lozano, J. (2005). L'estat actual del debat sobre la RSE a Espanya. En Responsabilitat Social a les Empreses. Symposium conducted at the meeting of Fundació Pau I Solidaritat, Barcelona.

Martínez González, J.A. (2011) Liderazgo y competencias directivas en época de crisis. Contribuciones a la Economía. Recuperable en http://www.eumed.net/ce/2011a/

McClelland, D. (1973). Testing for competence rather than inteligence. American Psychologist (46): 70-84

Muñoz, X. (2007). "La captación de recursos en las entidades de acción social". En Revista de Educación Social, 37, p. 101-116.

Navajo, P. (2009). Planificación estratégica en organizaciones no lucrativas. Madrid: Narcea.

Rodríguez Blanco, E., Carreras, I. y Sureda, M. (2012). Innovar para el cambio social. De la idea a la acción. Barcelona: Instituto de Innovación Social de ESADE.

Vernis, A., Iglesias, M., Sanz, B. y Saz, A. (2004). Los retos en la gestión de las organizaciones no lucrativas. Claves para el fortalecimiento institucional del tercer sector. Barcelona: Granica.

Vidal, P., Torres, D., Guix, B. y Peña, M. (2005). La responsabilitat social de les organitzacions no lucratives. Aproximació conceptual i desenvolupament del model RSO en Col-lecció Papers de Recerca OTS, 03. Observatori del Tercer Sector. 